\title{
Predictors of Posthepatectomy Ascites with or without Previous Portal Vein Embolization
}

\author{
Lisette T. Hoekstra $^{\mathrm{a}}$ Thijs Wakkie ${ }^{\mathrm{a}}$ Olivier R.C. Busch ${ }^{\mathrm{a}} \quad$ Dirk J. Gouma \\ Ulrich Beuers ${ }^{b}$ Thomas van Gulik ${ }^{\mathrm{a}}$ \\ Departments of aSurgery and ${ }^{\mathrm{b}}$ Hepatology, Academic Medical Center, Amsterdam, The Netherlands
}

\section{Key Words}

Ascites · Liver resection • Portal vein embolization •

Predictors

\begin{abstract}
Aim: To identify predictors of postoperative ascites after liver resection for patients with or without preoperative portal vein embolization (PVE). Methods: Patients undergoing PVE prior to hepatectomy (PVE group; $\mathrm{n}=37$ ) were compared with patients who underwent liver resection without PVE $(n=503)$. Ascites was defined as postoperative daily drainage of clear ascitic fluid exceeding $200 \mathrm{ml} /$ day. Pre-, intra-, and postoperative variables were retrospectively analyzed using uni- and multivariate analyses. Results: Postoperative ascites was present in $13.5 \%$ (5/37) of patients who underwent PVE before hepatectomy, compared to $5.8 \%(29 / 503)$ in the group undergoing liver resection without PVE $(p=0.061)$. In all patients, cirrhosis (OR 54.505, $\mathrm{p}<0.001$ ), operation time (OR 1.004, $p=0.014$ ), and the use of the Pringle maneuver (OR 2.336, $\mathrm{p}=0.041$ ) were independent risk predictors for ascites in multivariate analysis. In PVE patients, cirrhosis (OR
\end{abstract}

\section{KARGER}

Fax +4161306 1234

E-Mail karger@karger.ch

www.karger.com
(C) 2012 S. Karger AG, Basel

0253-4886/12/0296-0468\$38.00/0

Accessible online at:

www.karger.com/dsu
$0.156, p<0.001)$ was the only independent significant predictor of ascites after resection. In patients undergoing liver resection without $P V E$, independent risk factors with multivariate analysis were operation time $(\mathrm{OR} 1.005,=0.001)$ and cirrhosis (OR 26.609, $p<0.001$ ). Conclusion: Operation time and the use of the Pringle maneuver were significant predictors of ascites after hepatectomy. Cirrhosis was a significant risk factor associated with postoperative ascites.

Copyright $\odot 2012$ S. Karger AG, Basel

\section{Introduction}

Ascites is a common complication after liver resection with reported incidences ranging from 5 to $56 \%$ [1]. It may contribute to liver failure when large intra-abdominal ascitic fluid collections develop. Increased portal pressure after hepatectomy induced by a reduction in hepatic vascular bed leads to ascites production and subsequently decreased urinary output. Also, vasodilatation of the splanchnic vasculature promotes the formation of ascites [2]. Ascites is often seen in patients with preexisting 
cirrhosis and hepatocellular carcinoma (HCC) $[1,3-6]$ and may be aggravated after partial liver resection [7-9]. An important component in the formation of ascites in patients with HCC is microvascular hyperpermeability of tumor vessels due to HCC cells that secrete vascular endothelial growth factor. This results in extravasation of plasma and plasma proteins, and in accumulation of ascites fluid. With transection of the liver parenchyma in this patient group, the production of postoperative ascites is likely enhanced. Ascites may develop after liver resection irrespective of the quality of the parenchyma, making treatment strategies after liver resection difficult. To our knowledge, only one study has examined risk factors for ascites in patients with colorectal liver metastases (CRM) [10]. They reported that the Hx ratio (resected liver weight $\mathrm{g} /$ bodyweight $\mathrm{kg}$ ) was a significant independent factor for ascites after extended hepatectomy [10]. Other reported risk factors for ascites in patients with HCC are fibrosis [11], increased blood loss $(>1,000 \mathrm{ml})$, low platelet count $\left(<100 \times 10^{3} / \mu \mathrm{l}\right)$ [1], high indocyanine green retention rate $(>10 \%)$, hypoalbuminemia $(\leq 3.5 \mathrm{~g} /$ $\mathrm{dl}$ ), and extent of liver resection ( $\geq 3$ segments) [3].

Preoperative portal vein embolization (PVE) is a widely used method to increase resectability in patients with small-for-size future remnant liver, especially in patients with poor hepatic reserve. By increasing the volume and function of the future remnant liver preoperatively, the risk of liver failure after surgery is reduced. No studies have focused on the incidence of ascites after hepatectomy in patients also undergoing PVE. However, these patients might be more prone to develop postoperative complications since the initial tumor burden in patients requiring PVE is usually higher, the extent of liver resection usually greater, and consequently the future remnant liver smaller. The incidence and impact of postoperative ascites on the outcome in patients also undergoing PVE is uncertain.

The aims of this study were to examine the incidence and outcomes of ascites in patients undergoing liver resection with and without PVE, including a subgroup of patients with HCC, and to examine predictive factors for developing ascites after liver resection.

\section{Methods}

All 540 patients undergoing liver resection between January 1992 and February 2012 at the Academic Medical Center Amsterdam were analyzed. Of these patients, demographics, preoperative data, operative variables, presence or absence of ascites (volume, duration), morbidity, and mortality were evaluated retro- spectively. This group was then divided into patients who had undergone liver resection with $(n=37)$ or without PVE $(n=503)$. A subgroup of patients with HCC $(n=53)$ was also analyzed.

\section{Definitions}

All patients were reviewed in a multidisciplinary HPB team consisting of a liver surgeon, hepatologist, medical oncologist, gastroenterologist, and (interventional) radiologist. Future remnant liver volume was assessed by $\mathrm{CT}$ volumetry and hepatic function was determined by ${ }^{99 \mathrm{~m}} \mathrm{Tc}$-labelled mebrofenin hepatobiliary scintigraphy including SPECT preoperatively [12]. The volumes of total liver (TLV), tumor (TV), and future remnant liver (FRLV) were evaluated. The percentage of FRL was then calculated according to the following formula: FRLV $\times 100 /(T L V-T V)$. If the FRLV was $>25-30 \%$ in normal liver parenchyma, or $>40 \%$ in cirrhotic parenchyma (Child-Pugh A), the patient was considered eligible for surgery. Otherwise, PVE would be performed before liver resection. A cutoff value for future remnant liver function of $2.69 \% / \mathrm{min} / \mathrm{m}^{2}$ identified patients at risk of developing postoperative liver failure [13].

Major liver resections were defined as resections of three or more Couinaud segments. PVE is mostly performed when a right hemihepatectomy or extended right hemihepatectomy is planned, i.e. resection of 4 or 5 Couinaud segments. In our PVE group, all patients underwent right hemihepatectomy or extended right hemihepatectomy. Minor resections were hepatectomies of less than three liver segments, including wedge resections and metastasectomies. Prior to resection, all liver segments were systematically examined using intraoperative ultrasound to identify occult lesions. Parenchymal transection was performed using an ultrasonic dissection device (CUSA) with additional hemostasis using bipolar diathermy. Abdominal drainage was undertaken in all patients who underwent a major liver resection (3 or more segments). In patients who had a bisegmentectomy or (sub)segmentectomy, drains were only placed selectively.

Ascites was defined as postoperative daily drainage of clear ascitic fluid exceeding $200 \mathrm{ml} /$ day. We also recorded the duration (days) of postresectional ascites. The $\mathrm{Hx}$ ratio was calculated by dividing the weight of resected liver (in grams) by body weight (in kilograms) [10]. In-hospital mortality was defined as death during the entire postoperative admission.

\section{Management of Postoperative Ascites}

Conservative management of postoperative ascites consisted of fluid replacement. When albumin levels decreased $(<35 \mathrm{~g} / \mathrm{l})$, intravenous albumin was administered. Diuretic medication was administered in case of fluid retention. In patients with infected ascites, antibiotic treatment was added. Percutaneous drainage of the ascitic fluid was undertaken if intra-abdominal ascitic fluid collection was symptomatic, as identified by abdominal ultrasonography and/or CT scan. In case of persistence or increase in ascites formation, the abdominal drain was removed after the 5 th postoperative day. When discharge of ascitic fluid continued via the abdominal wall defect of the previous drain, surgical closure of the defect was performed.

\section{Study Population with HCC}

Standard diagnostic work-up of patients with HCC was performed in accordance with the guidelines of the AASLD [14]. This was especially true for lesions occurring in the background 
Table 1. Clinicopathological features of PVE patients with or without postoperative ascites after liver resection

\begin{tabular}{|c|c|c|c|}
\hline \multirow[t]{2}{*}{ Variables } & \multicolumn{3}{|l|}{$\operatorname{PVE}(\mathrm{n}=37)$} \\
\hline & no ascites & ascites & $\mathrm{p}$ \\
\hline Total, n (\%) & $32(86.5)$ & $5(13.5)$ & \\
\hline Mean age, years $\pm S D$ & $55.6 \pm 12.3$ & $64.2 \pm 7.8$ & 0.142 \\
\hline Male:female ratio & $20: 12$ & $4: 1$ & 0.452 \\
\hline Major:minor resections & $30: 2$ & $5: 0$ & 0.571 \\
\hline \multicolumn{4}{|l|}{ Diagnosis } \\
\hline CRM & 20 & 5 & 0.109 \\
\hline Klatskin & 5 & & \\
\hline Neuroendocrine tumor & 1 & & \\
\hline $\mathrm{HCC}$ & 1 & & \\
\hline Benign & 5 & & \\
\hline Median operation time, min (range) & $315(121-715)$ & $428(264-519)$ & 0.185 \\
\hline Cirrhosis, n (\%) & $0(0)$ & $0(0)$ & 1.000 \\
\hline Pringle maneuver, n (\%) & $6(18.8)$ & $3(60)$ & 0.148 \\
\hline Median time, min (range) & $20(20-60)$ & $30(20-40)$ & 0.477 \\
\hline Median time-period PVE and resection, days (range) & $38(22-435)$ & $29(19-33)$ & 0.031 \\
\hline
\end{tabular}

of cirrhosis. In general, liver resection was not indicated in patients with extrahepatic or nodal metastases, main portal trunk or inferior vena cava invasion or thrombus, or multicentric bilobar HCC. Most patients with Child-Pugh B and all patients with Child-Pugh $\mathrm{C}$ were excluded from resection.

\section{Statistical Analysis}

A two-tailed unpaired Student's t test was used for continuous parametric data for differences between groups. For nonparamateric data, the Mann-Whitney $U$ test was performed. Categorical data were compared using Fisher's exact test or $\chi^{2}$ test, where appropriate. Predictors of postoperative ascites were determined by univariate logistic regression analyses and subsequent multivariate logistic regression analyses. $\mathrm{p}<0.05$ was considered statistically significant. Statistical software (SPSS 18.0.0; SPSS, Chicago, Ill., USA) was used for the analysis.

\section{Results}

Data of all 540 patients who underwent a liver resection in our department are shown in figure 1. Overall, ascites production was recorded in 34 of the 540 patients (6.0\%). In patients who underwent PVE before hepatectomy, $13.5 \%$ (5/37) showed postoperative ascites, compared to $5.8 \%(29 / 503)$ in the group undergoing liver resection without PVE ( $\mathrm{p}=0.061)$. When analyzing the HCC subgroup, only 1 patient $(1 / 53,1.9 \%)$ had undergone preoperative PVE and did not show ascites production. In HCC patients that underwent liver resection without PVE, ascites occurred in 9 of the 52 patients (17.3\%; ns).
Table 2. Clinicopathological features of patients with or without postoperative ascites after liver resection without PVE

\begin{tabular}{lccr}
\hline Variables & \multicolumn{2}{c}{$\begin{array}{l}\text { Liver resection without PVE } \\
(\mathrm{n}=503)\end{array}$} & \\
\cline { 2 - 4 } & no ascites & ascites & $\mathrm{p}$ \\
\hline Total, n (\%) & $474(94.2)$ & $29(5.8)$ & \\
Mean age, years \pm SD & $54.4 \pm 15.3$ & $58.7 \pm 9.1$ & 0.204 \\
Male:female ratio & $207: 267$ & $18: 11$ & 0.053 \\
Major:minor resections & $205: 269$ & $198: 11$ & 0.048 \\
Diagnosis & & & \\
$\quad$ CRM & 195 & 10 & 0.183 \\
$\quad$ Klatskin & 35 & 6 & \\
$\quad$ Metastases & 23 & 1 & \\
$\quad$ HCC & 43 & 9 & \\
$\quad$ Benign & 163 & 3 & \\
$\quad$ Other & 15 & & 0.003 \\
Median operation time, & & & \\
$\quad$ min (range) & $230(39-756)$ & $310(115-719)$ & \\
Cirrhosis, n (\%) & $12(2.5)$ & $9(31.0)$ & $<0.001$ \\
Pringle maneuver, n (\%) & $122(25.7)$ & $12(41.4)$ & 0.070 \\
Median time, min (range) & $30(10-75)$ & $40(20-70)$ & 0.116 \\
\hline
\end{tabular}

Patient characteristics and operative data of all groups are depicted in tables 1 and 2 .

In the PVE group, there were significantly more males (64.9\%, 24/37 vs. 44.7\%, 225/503; $\mathrm{p}=0.018)$, more patients with CRM (67.6\%, 25/37 vs. 40.8\%, 205/503; p = 0.001), 
Table 3. Clinicopathological features of patients undergoing liver resection with or without previous PVE

\begin{tabular}{lccr}
\hline & PVE & No PVE & \multicolumn{1}{c}{$\mathrm{p}$} \\
\hline Total, n (\%) & $37(6.9)$ & $503(93.1)$ & \\
Mean age (SD), years & $56.8 \pm 12.1$ & $54.6 \pm 15.2$ & 0.461 \\
Male:female ratio & $24: 13$ & $225: 278$ & 0.018 \\
Major:minor resections & $35: 2$ & $223: 280$ & $<0.001$ \\
Diagnosis & & & \\
$\quad$ CRM & 25 & 205 & 0.001 \\
$\quad$ Klatskin & 5 & 47 & 0.407 \\
$\quad$ HCC & 1 & 52 & 0.132 \\
Median operation time, & & & \\
$\quad$ min (range) & $316(121-715)$ & $235(39-756)<0.001$ \\
Cirrhosis, n (\%) & $0(0)$ & $21(4.2)$ & 0.203 \\
Pringle maneuver, n (\%) & $9(24.3)$ & $134(26.6)$ & 0.546 \\
Median time, min (range) & $20(20-60)$ & $33(0-75)$ & 0.469 \\
Mortality, n (\%) & $2(5.4)$ & $14(2.8)$ & 0.365 \\
\hline
\end{tabular}

Table 4. Univariate and multivariate logistic regression analysis of factors associated with postoperative ascites

\begin{tabular}{lrcr}
\hline Factors & \multicolumn{1}{l}{ OR } & $95 \% \mathrm{CI}$ & $\mathrm{p}$ \\
\hline Univariate analysis & & & \\
Diagnosis & 0.905 & $0.829-0.988$ & 0.026 \\
Cirrhosis & 14.820 & $5.714-38.438$ & $<0.001$ \\
PVE & 2.554 & $1.023-7.042$ & 0.047 \\
Major resections & 2.411 & $1.151-5.051$ & 0.020 \\
Operation time & 1.004 & $1.001-1.006$ & 0.002 \\
Pringle maneuver & 2.245 & $1.108-4.550$ & 0.025 \\
\hline Multivariate analysis & & & \\
Cirrhosis & 54.505 & $14.048-211.467$ & $<0.001$ \\
Operation time & 1.004 & $1.001-1.007$ & 0.014 \\
Pringle maneuver & 2.336 & $1.033-5.278$ & 0.041 \\
\hline
\end{tabular}

more patients who had undergone more major resections (94.6\%, 35/37 vs. $44.3 \%, 223 / 503 ; \mathrm{p}<0.001)$, and longer operation time (median $316.0 \mathrm{~min}$, IQR: $121-715$ vs. 235.0 min, IQR: $39-756 ; \mathrm{p}<0.001)$ in comparison to patients undergoing liver resection without PVE. No significant differences were found for age, cirrhosis, application of the Pringle maneuver, or mortality. These outcomes are presented in table 3.

Overall, risk factors associated with ascites were diagnosis (HCC, Klatskin; OR 0.905, $\mathrm{p}=0.026$ ), cirrhosis (OR 14.820, $\mathrm{p}<0.001$ ), PVE (OR 2.554, $\mathrm{p}=0.047$ ), major resections (OR $2.411 \mathrm{p}=0.020)$, operation time (OR 1.004, $\mathrm{p}=0.002)$, and the use of the Pringle maneuver (OR
$2.245, \mathrm{p}=0.025)$, as determined by univariate analysis in all patients $(n=540)$. By subsequent multivariate analysis using these significant factors, cirrhosis (OR 54.505, $\mathrm{p}<$ 0.001 ), operation time (OR 1.004, $\mathrm{p}=0.014)$, and the use of the Pringle maneuver (OR 2.336, $\mathrm{p}=0.041$ ) were independent predictors of ascites postoperatively. These results are summarized in table 4 .

When separating the groups into PVE or not, cirrhosis (OR 0.156, p < 0.001) was the only independent significant predictor of ascites after resection in the PVE group. In patients undergoing liver resection without PVE, operation time (OR 1.003, $\mathrm{p}=0.010$ ), and cirrhosis (OR 17.325, $\mathrm{p}<0.001$ ) were significant risk factors for developing ascites after resection. Independent risk factors as calculated with multivariate analysis were operation time (OR 1.005, $\mathrm{p}=0.001$ ) and cirrhosis (OR 26.609, $\mathrm{p}<0.001)$.

In patients with ascites, the mean age was $64.2 \pm 7.8$ years in the PVE group in comparison to $58.7 \pm 9.1$ years in the liver resection group without PVE $(p=0.205)$. Also, the male:female ratio was not significantly different between the groups, nor was the diagnosis or the presence of cirrhosis. No differences were seen for type of resection, the Pringle maneuver, or operation time between both groups with postoperative ascites. Hospital stay was equal in patients with ascites who had undergone liver resection without previous PVE [median of 21 days (range: 7-79)] when compared with patients with ascites undergoing resection with PVE [median 15 days (range: 8-27); ns].

No significant differences were observed in the quantity or duration of ascites between the PVE group and group undergoing liver resection without PVE. Overall, the duration of postoperative ascites was less than 4 weeks in 28 patients $(68.3 \%)$. The median $\mathrm{Hx}$ ratio [resected liver weight $(\mathrm{g}) /$ bodyweight $(\mathrm{kg})$ ] was not different between groups with a value of 10.4 (range: 0.6-18.0) in patients who had undergone liver resection with PVE, and 11.6 (range 1.1-40.6) in patients without previous PVE. Treatment strategies are shown in figure 1.

In the HCC group $(n=53)$, only 1 patient underwent embolization of the portal vein. Mean age was $56.4 \pm 18.1$ years, with 17 HCC patients (32.1\%) showing cirrhosis. Ascites was present in $17.0 \%$ of the patients (9/53) including 8 patients (15.1\%) with cirrhosis. Major resections were performed in 21 patients, of whom 2 showed postoperative ascites. Median operation time was $242 \mathrm{~min}$ (range: 104-550). The median hospital stay was 11 days (range: 7-107). In-hospital mortality was 3.8\% $(\mathrm{n}=2)$. 


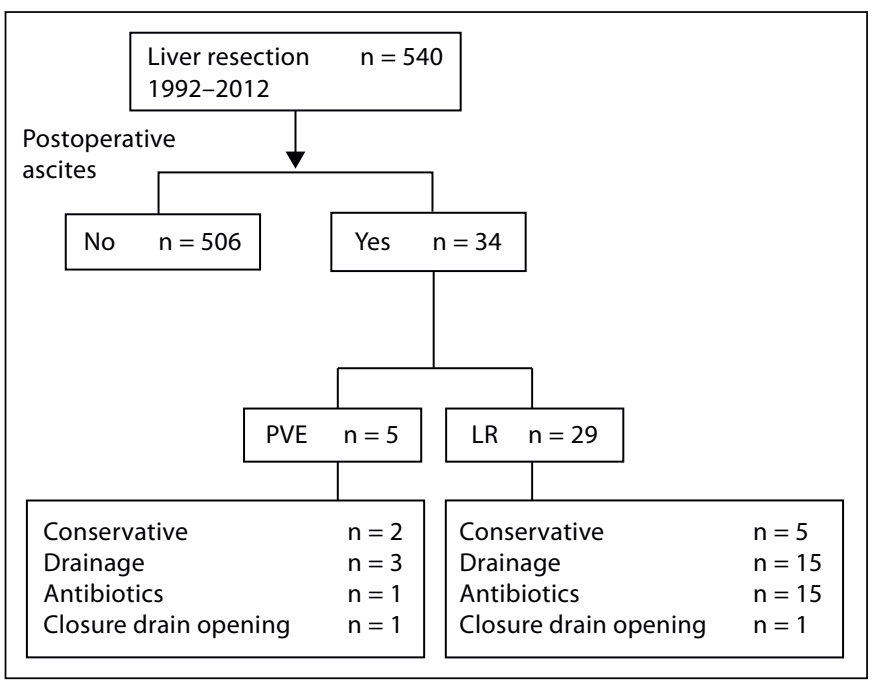

Fig. 1. Diagram of 540 patients who underwent liver resection (LR) between 1992 and 2012, showing postoperative ascites.

Table 5. Clinicopathological features of patients with or without cirrhosis

\begin{tabular}{lccr}
\hline & No cirrhosis & Cirrhosis & \multicolumn{1}{c}{$\mathrm{p}$} \\
\hline Total, n (\%) & $520(96.1)$ & $21(3.9)$ & \\
Mean age \pm SD, years & $54.7 \pm 15.1$ & $55.4 \pm 13.1$ & 0.832 \\
Male:female ratio & $235: 285$ & $15: 6$ & 0.018 \\
PVE, n (\%) & $37(7.1)$ & $0(0)$ & 0.206 \\
Major:minor resections & $257: 263$ & $2: 19$ & $<0.001$ \\
Diagnosis & & & \\
$\quad$ CRM & 230 & 0 & $<0.001$ \\
$\quad$ Klatskin & 52 & 0 & 0.128 \\
$\quad$ HCC & 36 & 17 & $<0.001$ \\
Median operation time, & & & \\
$\quad$ min (range) & $244(39-756)$ & $214(69-327)$ & 0.051 \\
Pringle maneuver, n (\%) & $140(26.9)$ & $3(14.3)$ & 0.176 \\
Median time, min (range) & $30(0-75)$ & $30(0-60)$ & 0.738 \\
Ascites, n (\%) & $25(4.8)$ & $9(42.9)$ & $<0.001$ \\
Mortality, n (\%) & $16(3.1)$ & $0(0)$ & 0.415 \\
\hline & & & \\
\hline
\end{tabular}

Data of patients with or without cirrhosis are shown in table 5.

\section{Discussion}

Although several published studies have analyzed the risk factors in patients with HCC and ascites, the influence of postoperative ascites associated with PVE in pa- tients undergoing liver resection are not clear. It is anticipated that the presence of ascites may impact the outcome of these patients; however, to the best of our knowledge, this is the first large study examining risk factors for the development of ascites after hepatectomy in patients undergoing preoperative PVE or not.

One might hypothesize that PVE before surgery would reduce the risk of ascites postoperatively because the main portal flow through the liver remnant is unaffected after right hemihepatectomy and previous occlusion of the right portal vein; however, our findings showed a higher incidence of ascites in the PVE group after resection ( 13.5 vs. $5.8 \%)$. This is probably related to the larger resections required in patients who had undergone PVE preoperatively, as is supported by our findings that patients with hepatectomies of 3 or more segments had a higher incidence of ascites (OR 2.411), while more major resections were performed in the PVE group (i.e. 94.6 and $44.3 \%$, respectively, $\mathrm{p}<0.001$ ). Besides type of resection, the operation time was longer in the PVE group compared to the patients undergoing liver resection without PVE. PVE is especially performed in patients requiring extended resections, which are commonly associated with longer operations times.

As mentioned above, patients undergoing PVE before hepatectomy are a highly selected group of patients which will undergo major, even critically extended resections, compared to a normal cohort of hepatectomy patients (as in this study in which $94.6 \%$ of patients in the PVE group had undergone major resections, whereas in the non-PVE group, $44.3 \%$ of patients had major resections). Therefore, the outcome of PVE in patients all undergoing the same type of major resections was also analyzed, and it showed that the only risk factor associated with ascites was diagnosis (OR 0.857, $\mathrm{p}=0.047)$ in all patients $(\mathrm{n}=$ 258 ) as determined by univariate analysis. When separating the groups into PVE and non-PVE, no significant independent predictor of ascites after resection was found in the PVE group or in the patients undergoing liver resection without PVE.

In the literature, incidences of $5-56 \%$ have been reported for postoperative ascites $[1,15]$. In our study, $6.0 \%$ $(34 / 540)$ of all patients showed ascitic fluid production after liver resection, which is in accordance with the more recent reports of postresectional ascites $[3,16,17]$. The decrease in postresectional ascites in more recent years is possibly due to improved perioperative fluid management. The underlying mechanisms of ascites formation after hepatectomy are, however, largely unknown. 
When analyzing all patients, we also found by multivariate analysis that cirrhosis was a risk factor for developing postoperative ascites (OR 54.505). In a recent study, Chan et al. [3] showed that ascites developed in $25.5 \%$ of patients with HCC undergoing liver resection and that cirrhosis was present in $70.6 \%$ of all patients. In our HCC study population, $17.0 \%(9 / 53)$ of patients showed postresectional ascites, and $32.1 \%(17 / 53)$ of the patients had a cirrhotic liver based on histopathological evaluation of the resection specimens.

We realize that more malignant lesions such as CRM and Klatskin tumors were present in patients undergoing PVE before hepatectomy in comparison to more benign liver lesions in the resection group without PVE, resulting in a possible bias. However, no significant difference in the incidence of ascites was observed among patients after resection of malignant or benign tumors, rendering any influence unlikely.

No official definition of postoperative ascites is available, but a grading system has been proposed by the International Ascites Club [7] for patients with cirrhotic livers. According to this system, mild ascites, which is only detectable by ultrasonography, corresponds with grade I. Grade II is defined as moderate ascites manifested by moderate symmetrical distension of the abdomen, and grade III is large or gross ascites with abdominal distension. In as much as the validity of this classification system has not been shown in patients with ascites after liver resection, we decided to define ascites production as the drainage of $200 \mathrm{ml} /$ day or more of ascitic fluid in this study. In our study, a pigtail drain was percutaneously placed in the abdomen under ultrasound guidance for drainage in case of ascites. However, an important remark is that our definition may have underestimated the real number of patients with postoperative ascites since our definition did not include patients in whom ascites drained spontaneously via the laparotomy wound. Furthermore, in some patients the amount of ascites was unknown because the ascitic fluid did not require drainage. We assume, however, that in these patients the ascitic fluid did not exceed $200 \mathrm{ml} /$ day as proposed in the definition. Some patients were discharged from the hospital with ascites shown on abdominal imaging studies without needing drainage. Again, we do not believe that these fluid collections were clinically relevant and did not exceed $200 \mathrm{ml} /$ day. Percutaneous drainage of the ascitic fluid was only undertaken if intra-abdominal fluid collection was symptomatic, as confirmed by abdominal ultrasound and/or CT scan.

In conclusion, our analysis showed that operation time and the use of the Pringle maneuver were significant predictors of ascites after hepatectomy. A significant association was found between cirrhosis and the occurrence of postresectional ascites.

\section{Disclosure Statement}

There is no conflict of interest to disclose or funding sources to declare.

\section{References}

1 Ishizawa T, Hasegawa K, Kokudo N, Sano K, Imamura H, Beck Y, Sugawara Y, Makuuchi M: Risk factors and management of ascites after liver resection to treat hepatocellular carcinoma. Arch Surg 2009;144:46-51.

2 Buob S, Johnston AN, Webster CR: Portal hypertension: pathophysiology, diagnosis, and treatment. J Vet Intern Med 2011;25: 169-186.

-3 Chan KM, Lee CF, Wu TJ, Chou HS, Yu MC, Lee WC, Chen MF: Adverse outcomes in patients with postoperative ascites after liver resection for hepatocellular carcinoma. World J Surg 2012;36:392-400.

4 Ikeda Y, Kanematsu T, Matsumata T, Shimada M, Yamagata M, Sugimachi K: Liver resection and intractable postoperative ascites. Hepatogastroenterology 1993;40:14-16.
5 Moinuddin M, Rockett JF, Waber A: Letter: Scanning for pulmonary calcification. Ann Intern Med 1976;84:224-225.

6 Shimada M, Takenaka K, Fujiwara Y, Gion T, Shirabe K, Yanaga K, Sugimachi K: Risk factors linked to postoperative morbidity in patients with hepatocellular carcinoma. $\mathrm{Br}$ J Surg 1998;85:195-198.

7 Moore KP, Wong F, Gines P, Bernardi M, Ochs A, Salerno F, Angeli P, Porayko M, Moreau R, Garcia-Tsao G, Jimenez W, Planas $\mathrm{R}$, Arroyo V: The management of ascites in cirrhosis: report on the consensus conference of the International Ascites Club. Hepatology 2003;38:258-266.

8 Runyon BA: Care of patients with ascites. N Engl J Med 1994;330:337-342.

$\checkmark 9$ Runyon BA: Management of adult patients with ascites due to cirrhosis: an update. Hepatology 2009;49:2087-2107.
10 Shimizu Y, Sano T, Yasui K: Predicting pleural effusion and ascites following extended hepatectomy in the non-cirrhotic liver. J Gastroenterol Hepatol 2007;22:837-840.

11 Farges O, Malassagne B, Flejou JF, Balzan S, Sauvanet A, Belghiti J: Risk of major liver resection in patients with underlying chronic liver disease: a reappraisal. Ann Surg 1999; 229:210-215.

12 de GW, van Lienden KP, van Gulik TM, Bennink RJ: (99m)Tc-mebrofenin hepatobiliary scintigraphy with SPECT for the assessment of hepatic function and liver functional volume before partial hepatectomy. J Nucl Med 2010;51:229-236. 
13 de Graaf W, van Lienden KP, Dinant S, Roelofs JJ, Busch OR, Gouma DJ, Bennink RJ, van Gulik TM: Assessment of future remnant liver function using hepatobiliary scintigraphy in patients undergoing major liver resection. J Gastrointest Surg 2010;14:369-378.

14 Bruix J, Sherman M: Management of hepatocellular carcinoma. Hepatology 2005;42: 1208-1236.
15 Fuster J, Garcia-Valdecasas JC, Grande L, Tabet J, Bruix J, Anglada T, Taura P, Lacy AM, Gonzalez X, Vilana R, Bru C, Sole M, Visa J: Hepatocellular carcinoma and cirrhosis. Results of surgical treatment in a European series. Ann Surg 1996;223:297-302.

16 Chen MS, Li JQ, Zheng Y, Guo RP, Liang HH, Zhang YQ, Lin XJ, Lau WY: A prospective randomized trial comparing percutaneous local ablative therapy and partial hepatectomy for small hepatocellular carcinoma. Ann Surg 2006;243:321-328.
17 Harada N, Shirabe K, Jjichi H, Matono R, Uchiyama $\mathrm{H}$, Yoshizumi T, Taketomi A, Soejima Y, Maehara Y: Acoustic radiation force impulse imaging predicts postoperative ascites resulting from curative hepatic resection for hepatocellular carcinoma. Surgery 2012 . 